\title{
Surgical treatment of secondary mitral regurgitation: is repair a reasonable option?
}

\author{
Clarence Pingpoh, Matthias Siepe, Wolfgang Bothe \\ Department of Cardiovascular Surgery, University Heart Center Freiburg-Bad Krozingen, Faculty of Medicine, University of Freiburg, Freiburg, \\ Germany \\ Contributions: (I) Conception and design: All authors; (II) Administrative support: M Siepe, W Bothe; (III) Provision of study materials or patients: \\ All authors; (IV) Collection and assembly of data: C Pingpoh, W Bothe; (V) Data analysis and interpretation: C Pingpoh, M Siepe; (VI) Manuscript \\ writing: All authors; (VII) Final approval of manuscript: All authors. \\ Correspondence to: Clarence Pingpoh, MD. Department of Cardiovascular Surgery, University Heart Center Freiburg-Bad Krozingen, Hugstetter \\ Strasse 55, 79106 Freiburg, Germany. Email: Clarence.Pingpoh@universitaets-herzzentrum.de.
}

Background: The optimal approach for treating secondary mitral regurgitation (MR) is debated. Since
annuloplasty alone has resulted in $30 \%$ or more recurrent $\mathrm{MR}$ in the mid-term, recent studies suggest mitral valve
replacement. However, subvalvular repair strategies as an adjunct to annuloplasty in order to prevent recurrent
MR due to ongoing left ventricle (LV) remodeling are becoming increasingly popular. In this study we present
short term results of patients with secondary MR who underwent mitral repair using a novel subvalvular repair
approach and address the questions whether mitral repair is a reasonable option in these patients. Methods: Ten patients presenting with functional MR underwent ring annuloplasty and a subvalvular approximation of the papillary muscles using a technique where the distance between both papillary muscles was adjusted with a Polytetrafluoroethylene (PTFE) string during the saline test (Ring-Noose-String technique).

Results: Nine of ten patients had none-mild MR while in one patient mild-moderate MR due to ongoing leaflet tethering was diagnosed at discharge. In this patient, insufficient adjustment of the PTFE string is hypothesized as the primary mechanism for suboptimal repair. There were no complications related to the novel surgical technique. No patient died during the follow up period.

Conclusions: The Ring-Noose-String technique provides acceptable repair results in the short term. A longer follow-up is needed to proof its long-term efficacy. Subvalvular techniques such as this may allow a durable repair in patients with secondary MR.

Keywords: Functional mitral valve regurgitation; ring noose string technique; papillary muscle approximation

Received: 16 August 2017; Accepted: 11 September 2017; Published: 07 November 2017.

doi: 10.21037 /jovs.2017.09.09

View this article at: http://dx.doi.org/10.21037/jovs.2017.09.09

\section{Introduction}

The main components of the mitral valve are the annulus, the leaflets, the papillary muscles and the chordae tendineae. The ideal functioning of the mitral valve complex is dependent on the smooth interaction between the left ventricular wall and the 4 components mentioned above (1).

Current guidelines categorize mitral regurgitation (MR) as either primary (organic) or secondary (ischemic and functional) in nature $(1,2)$. Primary MR is caused by a structural defect or damage of one of the mitral valve components, while secondary MR is caused by a left ventricular dilation resulting in an expansion of the mitral annulus and lateral displacement of the papillary muscles leading to valve insufficiency $(3,4)$. In such cases, the remodeled left ventricle (LV) is markedly enlarged and the resulting MR is due to tethering of the subvalvular apparatus leading to restriction of the mitral leaflets $(1,4)$. The causes of this LV remodelling are either regional, as is the case with ischemia or global, which may be either an 
Table 1 Baseline characteristics

\begin{tabular}{lc}
\hline Baseline characteristics & $\mathrm{N}=10$ \\
\hline Age (years) & $56.4[37-76]$ \\
Male & $8[80]$ \\
Arterial hypertension & $4[40]$ \\
Atrial fibrillation & $5[50]$ \\
LVEDD (mm) & $63.1[57-67]$ \\
Dilative cardiomyopathy & $6[60]$ \\
Ischemic cardiomyopathy & $4[40]$ \\
Coronary vessel disease & $4[40]$ \\
Left ventricular ejection fraction & \\
$40-54 \%$ & $1[10]$ \\
$30-39 \%$ & $5[50]$ \\
$<30 \%$ & $4[40]$ \\
NYHA class III or IV & $10[100]$
\end{tabular}

Data are shown as number (\%) or median (range). LVEDD, left ventricular end diastolic diameter; NYHA, New York Heart Association.

idiopathic or dilated cardiomyopathy, resulting in a change in $L V$ geometry causing severe $L V$ dysfunction.

Hence the main pathophysiological mechanisms of secondary MR are increased tethering forces caused by papillary muscle displacement in the enlarged left ventricle as well as more apical placement of the leaflets and leaflet coaptation point $(1,4)$.

The therapy option for functional MR is debatable as the crux of the matter lies in the geometry of the $\mathrm{LV}$ and not in the mitral valve itself. Hence it is less obvious whether the isolated correction of the MR without altering the LV geometry will be remedial (5-8). Current guidelines do not provide guidance in selecting an adequate treatment approach; hence controversy exists as regards repair or replacement (2). Isolated mitral valve repair with a restrictive rigid annuloplasty has been associated with equal or better remodeling and mortality rates (6-8), but higher MR recurrence rates have been observed when comparing repair to replacement $(6,8)$. The main reason of the MR recurrence is the displacement of the posterior annulus anteriorly by the annuloplasty ring resulting in an augmentation of the posterior leaflet tethering. In order to adequately address this, both the mitral valve annulus and the subvalvular apparatus need to be attended to. The American Association of Thoracic Surgery consensus guidelines suggests that mitral valve replacement should be the treatment of choice for patients with a high risk for postoperative MR recurrence after simple restrictive annuloplasty (such as functional MR) and for those undergoing surgery in less-experienced centers where annuloplasty alone is the only repair option offered (2). In this article we present our treatment technique for this problem as well as initial results of the subvalvular mitral valve repair with the ring-noose technique.

\section{Patient selection and workup}

The patients were referred to our institution with a clinical presentation of symptomatic MR and eventually LV dysfunction (Table 1). The diagnosis and severity of the MR was confirmed after physical examination and transesophageal echocardiography. All 10 patients had at least a moderate to severe left ventricular dysfunction and had symptoms of heart failure or pulmonary oedema prior to surgery. A written informed consent from the patients for publication of this article and its accompanying images are available.

\section{Pre-operative preparation}

Preoperative transesophageal echocardiography was performed in each patient. Functional MR was defined by MR due to leaflet tethering (posterior leaflet angle $\geq 45^{\circ}$, tenting height above $1 \mathrm{~cm}$ ) and moderate to severe $\mathrm{LV}$ dysfunction [ejection fraction (EF) $<40 \%$ )] and cardiac catheterization was also performed in order to assess the coronary arteries. The carotid arteries were examined using duplex sonography. Occasionally we also do a computed tomography of the ascending aorta to enable us to decide whether or not do a minimal invasive procedure.

\section{Equipment preference card}

The equipment includes the standard setting for cardiac surgery with a heart lung machine. The repair can be performed either as a minimal invasive procedure through a right anterolateral thoracotomy or through full sternotomy. Besides the basic equipment, a $3 \mathrm{~mm}$ in diameter felt-enforced 3-CV polytetrafluoroethylene (PTFE) noose as well as a double-armed, felt-enforced 3-CV PTFE string and a complete annuloplasty ring are needed for the ring noose technique. 


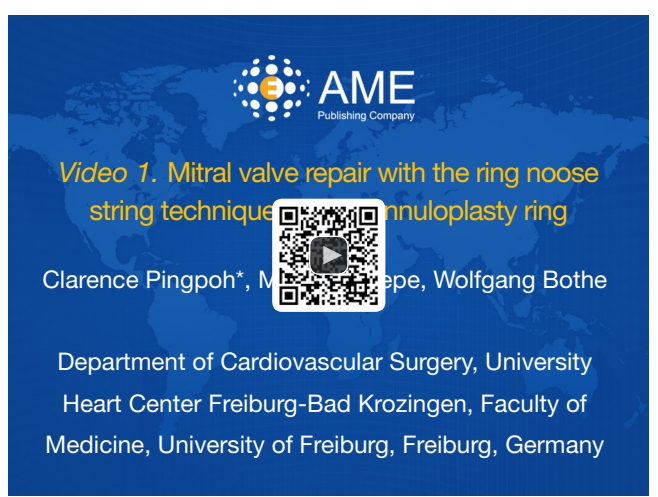

Figure 1 Mitral valve repair with the ring noose string technique and an annuloplasty ring (9).

Available online: http://www.asvide.com/articles/1817

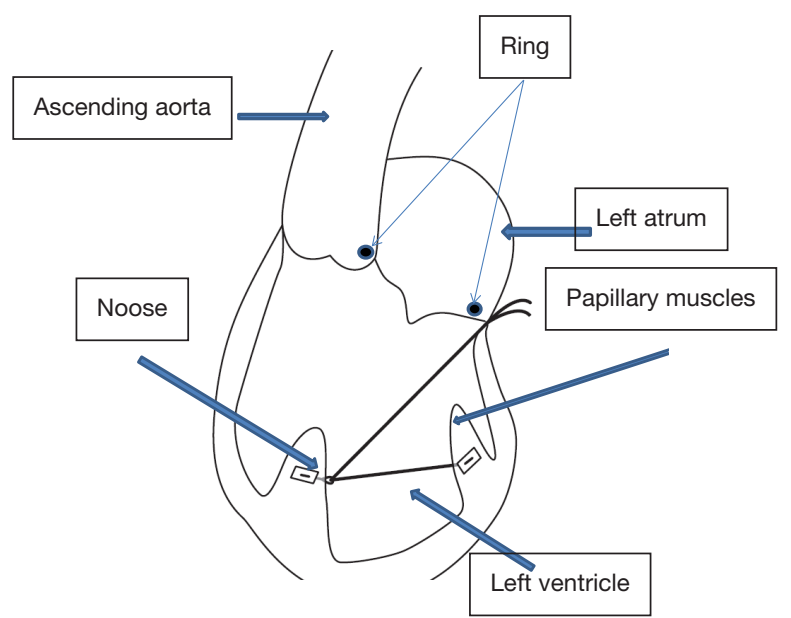

Figure 2 Schematic illustration of the Ring noose technique.

\section{Procedure}

\section{Mitral valve repair with the Ring Noose String Technique}

The procedure may be performed either through a full sternotomy or through a minimally invasive right anterior thoracotomy. Cardiopulmonary bypass is established in a standardized fashion. Exposure to the mitral valve is obtained through the interatrial groove. Retractors are positioned at 1 and 4 o'clock to ease exposure. A Dubost incision or transseptal approach may be considered for very small atria or repeat operations.

Ring sutures are then placed around the mitral annulus and the papillary muscles are identified. A feltenforced 3-CV PTFE noose is fastened at the base of the anterolateral papillary muscle and a double-armed, felt- enforced 3-CV PTFE string is sewn to the bottom of the posteromedial papillary muscle. The ends of the PTFE string are guided through the noose, so that pulling on PTFE string results in an approximation of the papillary muscles (Figure 1). The string is externalized in the left atrium through the mid-posterior mitral annulus behind the P2 segment (Figure 2). A complete, downsized annuloplasty ring is implanted and the ends of the PTFE string are loosely stitched through the ring. The saline test is performed and the string ends are attached and fixed to the annuloplasty ring when coaptation point is optimal.

\section{Role of team members}

* The cardiologist performs the preoperative workup with echocardiography and cardiac catheterization;

* The surgeon performs the procedure together with the trainee;

* The trainee assists the surgeon throughout the procedure;

* The anesthetist takes care of the intravenous anesthesia during the procedure as well as the pain management afterwards. Importantly, the anesthesiologist performs the intraoperative transesophageal echocardiography. They are also present in the treatment of the patient on the ICU after surgery;

* The perfusionist is in charge of the heart lung machine throughout the procedure;

* The theater nurse assists the surgeon and the trainee during the procedure;

* The ICU nurse assists the physicians on the ICU;

* The physiotherapist makes sure the patient is timely mobilized.

\section{Post-operative management}

After surgery the patients are transferred to the intensive care unit (ICU). As soon as vital and metabolic functions are stabilized and there is no bleeding, the patient is allowed to wake up and is then taken off the ventilator. In routine cases, this procedure begins few hours after surgery. Majority of the patients are maintained at the ICU for 24 to 48 hours after which they will be transferred to an intermediate care unit. Most patients are discharged within 8 to 12 days after surgery.

\section{Post-operative day 1 and 2}

Hemodynamic, respiratory, metabolic and nutritional 
Table 2 Perioperative data

\begin{tabular}{|c|c|c|c|}
\hline \multirow[t]{2}{*}{ Patient } & \multirow[t]{2}{*}{ Mitral valve pathology } & \multicolumn{2}{|c|}{$\begin{array}{l}\text { Mitral valve regurgitation } \\
\text { grade }\end{array}$} \\
\hline & & Preoperative & Postoperative \\
\hline 1 & Annular dilation & 3 & 1 \\
\hline 2 & Annular dilation & 3 & 1 \\
\hline 3 & Annular dilation + tethering & 3 & 1 \\
\hline 4 & $\begin{array}{c}\text { Annular dilation + P2 } \\
\text { prolapse }\end{array}$ & 3 & 1 \\
\hline 5 & Annular dilation + tethering & 3 & 1 \\
\hline 6 & Annular dilation & 3 & 1 \\
\hline 7 & Annular dilation + tethering & 2 & 1 \\
\hline 8 & $\begin{array}{l}\text { Annular dilation + posterior } \\
\text { prolapse }\end{array}$ & 3 & 0 \\
\hline 9 & Annular dilation & 3 & 2 \\
\hline 10 & Annular dilation + tethering & 3 & 1 \\
\hline
\end{tabular}

aspects of recovery are painstakingly monitored and managed as needed by the patient.

\section{Post-operative day 3 to 5}

Hemodynamic, respiratory, metabolic and nutritional treatments are maintained, adjusted, or abated as medically needed. The surgical team is involved on a daily basis in the decision making process. The laboratory tests, chest X-ray and echocardiography will be reviewed by the care team while anticipating discharge.

\section{Discharge and postoperative follow up}

Echocardiographic follow up at 12 weeks, 6 months and thereafter yearly.

\section{Tips, tricks and pitfalls}

* Attention is required to avoid tangling of the PTFE strings with primary chordae while stitching it through the mitral annulus;

* The most lateral and the most septal head of the respective papillary muscle should be chosen to maximize the ability of decreasing the interpapillary muscle distance;

* Choosing the right length of the string between the papillary muscles is crucial, but may vary and is not easily standardized. It is hypothesized that the length of the PTFE string was chosen too long in the patient with mild-moderate postoperative MR grades, thus leading to ongoing leaflet tethering.

\section{Conclusions}

The approach of treating secondary MR surgically by repair or replacement continues to be debated, since annuloplasty alone has resulted in $30 \%$ or more recurrent MR in the mid-term. The Ring-Noose-String technique provides a mechanism-oriented adjunct repair approach with acceptable results in the short term (Table 2). A longer follow-up is needed to proof its long-term efficacy. Subvalvular techniques such as this may allow a durable repair in patients with secondary MR.

\section{Acknowledgements}

None.

\section{Footnote}

Conflicts of Interest: The authors have no conflicts of interest to declare.

Ethical Statement: The study has been submitted to the ethics committee of the University of Freiburg (Germany) and written informed consent was obtained from all patients.

\section{References}

1. 1. Piérard LA, Carabello BA. Ischaemic mitral regurgitation: pathophysiology, outcomes and the conundrum of treatment. Eur Heart J 2010;31:2996-3005.

2. Nishimura RA, Otto CM, Bonow RO, et al. 2014 AHA/ ACC guideline for the management of patients with valvular heart disease: executive summary: a report of the American College of Cardiology/American Heart Association Task Force on Practice Guidelines. J Am Coll Cardiol 2014;63:2438-88.

3. Yiu SF, Enriquez-Sarano M, Tribouilloy C, et al. Determination of the degree of functional mitral regurgitation in patients with systolic left ventricular dysfunction: a quantitative clinical study. Circulation 2000;102:1400-6. 
4. He S, Fontaine AA, Schwammenthal E, et al. Integrated mechanism for functional mitral regurgitation: leaflet restriction versus coapting force: in vitro studies. Circulation 1997;96:1826-34.

5. Ciarka A, Braun J, Delgado V, et al. Predictors of mitral regurgitation recurrence in patients with heart failure undergoing mitral valve annuloplasty. Am J Cardiol 2010;106:395-401.

6. Goldstein D, Moskowitz AJ, Gelijns AC, et al. CTSN. Two-year outcomes of surgical treatment of severe ischemic mitral regurgitation. $\mathrm{N}$ Engl J Med 2016;374:344-53.

doi: 10.21037/jovs.2017.09.09

Cite this article as: Pingpoh C, Siepe M, Bothe W. Surgical treatment of secondary mitral regurgitation: is repair a reasonable option? J Vis Surg 2017;3:158.
7. Acker MA, Parides MK, Perrault LP, et al. Mitral-valve repair versus replacement for severe ischemic mitral regurgitation. N Engl J Med 2014;370:23-32

8. Calafiore AM, Gallina S, Di Mauro M, et al. Mitral valve procedure in dilated cardiomyopathy: repair or replacement? Ann Thorac Surg 2001;71:1146-52; discussion 1152-3.

9. Pingpoh C, Siepe M, Bothe W. Mitral valve repair with the ring noose string technique and an annuloplasty ring. Asvide 2017;4:498. Available online: http://www.asvide. com/articles/1817 